\title{
Lightweight Block Ciphers Revisited: Cryptanalysis of Reduced Round PRESENT and HIGHT
}

\author{
Onur Özen ${ }^{1}$, Kerem Varıc1 ${ }^{2}$, Cihangir Tezcan ${ }^{3}$, and Çelebi Kocair ${ }^{4}$ \\ 1 EPFL IC LACAL Station 14. CH-1015 Lausanne, Switzerland \\ onur.ozen@epfl.ch \\ 2 K.U.Leuven, Dept. of Electrical Engineering, ESAT/SCD/COSIC and IBBT \\ Kasteelpark Arenberg 10, B-3001 Heverlee, Belgium \\ kerem.varici@esat.kuleuven. be \\ 3 METU, Institute of Applied Mathematics, Department of Cryptography. 06531 \\ Ankara, Turkey \\ cihangir@metu.edu.tr \\ 4 METU, Department of Computer Engineering. 06531 Ankara, Turkey \\ celebi@ceng.metu.edu.tr
}

\begin{abstract}
Design and analysis of lightweight block ciphers have become more popular due to the fact that the future use of block ciphers in ubiquitous devices is generally assumed to be extensive. In this respect, several lightweight block ciphers are designed, of which PREsEnT and HigHT are two recently proposed ones by Bogdanov et al. and Hong et al. respectively. In this paper, we propose new attacks on PRESENT and Hight. Firstly, we present the first related-key cryptanalysis of 128bit keyed PRESENT by introducing 17-round related-key rectangle attack with time complexity approximately $2^{104}$ memory accesses. Moreover, we further analyze the resistance of HiGHT against impossible differential attacks by mounting new 26-round impossible differential and 31-round related-key impossible differential attacks where the former requires time complexity of $2^{119.53}$ reduced round HiGHT evaluations and the latter is slightly better than exhaustive search.
\end{abstract}

Keywords : Present, Hight, Related-Key Attack, Rectangle Attack, Impossible Differential Attack.

\section{Introduction}

Lightweight cryptography has become very vital with the emerging needs in sensitive applications like RFID (Radio-frequency identification) systems and

\footnotetext{
* This work was sponsored by the Research Fund K.U.Leuven, by the IAP Programme P6/26 BCRYPT of the Belgian State (Belgian Science Policy) and by the European Commission through the ICT Programme under Contract ICT-2007-216676 (ECRYPT II). The information in this paper is provided as is, and no warranty is given or implied that the information is fit for any particular purpose. The user thereof uses the information at its sole risk and liability.
} 
sensor networks. For these types of special purposes, there is a strong demand in designing secure lightweight cryptographic modules. After the selection of AES (Advanced Encryption Standard) [1, the research on efficient implementation of AES, especially for such constrained environments, brought special attention in research community. Even though it is highly convenient for such devices, the research on designing and analyzing new lightweight block ciphers that are more efficient than AES on these platforms poses huge challenges. For this purpose, several block ciphers are designed as potential candidates such as Hight [2]3, Present [4, mCrypton [5], SEA [6], CGEN [7], DES [8] and DESXL [8 ${ }^{5}$

A recent portfolid 6 which contains four software and three hardware oriented stream ciphers [11, has been announced by ECRYPT as part of eSTREAM project to identify new stream ciphers that might become suitable for widespread adoption including lightweight platforms. As a result, stream ciphers are shown to be highly efficient on both software and hardware implementations comparing to block ciphers. To fill this efficiency gap, Present [4] was proposed by Bogdanov et al. at CHES ' 07 as an ultra-lightweight block cipher with 31 rounds offering as good hardware and software performance as current modern stream ciphers while it is more efficient than many known block ciphers.

Basic security analysis of PRESENT is provided in [4 by showing resistance against known attacks such as differential, linear cryptanalysis and their variants. Recent differential attacks [12]13] on 16 and 19 rounds of PRESENT provide similar results as in the original proposal with some practical evidence of applied characteristics where the latter is an attempt to combine algebraic attacks with differential cryptanalysis. Another type of an attack called bit-pattern based integral attack [14] is applicable up to seven rounds of PRESENT. More recently, a new type of attack, called statistical saturation attack was proposed in [15] and shown to be applicable up to 24 rounds of PrEsent. Previous results on the analysis of PRESENT are summarized in Table 1.

The security of PRESENT against key schedule weaknesses is provided by showing the resistance against slide [16] and related-key differential attacks [17] where slide attacks are inapplicable because of the round dependent counters in key scheduling algorithm. Related-key differential attacks, on the other hand, are also believed to be inapplicable because of the sufficient non-linearity due to key scheduling algorithm.

Hight [2]3 is a South Korean standard encryption algorithm enjoying the use of a low-resource hardware implementation. It is a 32 round block cipher proposed one year before PRESENT at CHES '06 by Hong et al. to be used for ubiquitous computing devices. The prominent characteristic of Hight is that it makes use of simple byte oriented operations such as exclusive-or, addition modulo 256 and cyclic rotation which offers nice performance on hardware.

${ }^{5}$ TEA [9] and XTEA [10] can also be given as lightweight block ciphers which were designed before AES.

${ }^{6}$ The original hardware-oriented portfolio of eSTREAM contains four hardwareoriented stream ciphers. However, F-FSCR-H has recently been eliminated from the eSTREAM portfolio. 
Table 1. Summary of the attacks on Present and Hight (CP-Chosen Plaintext, MA-Memory Accesses, PR-Reduced round Present evaluation, HEReduced round Hight evaluation)

\begin{tabular}{|c|c|c|c|c|c|c|c|}
\hline Cipher & Rounds & $\begin{array}{c}\text { Key } \\
\text { Size }\end{array}$ & $\begin{array}{c}\text { Attack } \\
\text { Type }\end{array}$ & $\begin{array}{c}\text { Data } \\
\text { Complexity }\end{array}$ & $\begin{array}{c}\text { Time } \\
\text { Complexity }\end{array}$ & $\begin{array}{c}\text { Memory } \\
\text { Complexity }\end{array}$ & Reference \\
\hline PRESENT & 24 & 80 & Stat. Sat. & $2^{60} \mathrm{CP}$ & $2^{20} \mathrm{PR}$ & $2^{16}$ bytes & {$[15$} \\
\hline & 24 & 80 & Stat. Sat. & $2^{57} \mathrm{CP}$ & $2^{57} \mathrm{PR}$ & $2^{32}$ bytes & {$[15$} \\
\hline & 7 & 128 & Bit-Pat. Int. & $2^{24.3} \mathrm{CP}$ & $2^{100.1} \mathrm{MA}$ & $2^{77}$ bytes & 14 \\
\hline & 17 & 128 & Rel.-Key Rec. & $2^{63} \mathrm{CP}$ & $2^{104} \mathrm{MA}$ & $2^{53}$ bytes & $\vdots 3.1$ \\
\hline & 19 & 128 & Alg.-Dif. & $6 \times 2^{62} \mathrm{CP}$ & $2^{113} \mathrm{MA}$ & not specified & 13 \\
\hline HIGHT & 18 & 128 & Imp. Dif. & $2^{46.8} \mathrm{CP}$ & $2^{109.2} \mathrm{HE}$ & not specified & {$[2$} \\
\hline & 25 & 128 & Imp. Dif. & $2^{60} \mathrm{CP}$ & $2^{126.78} \mathrm{HE}$ & not specified & 18 \\
\hline & 26 & 128 & Imp. Dif. & $2^{61} \mathrm{CP}$ & $2^{119.53} \mathrm{HE}$ & $2^{109}$ bytes & 4.1 \\
\hline & 26 & 128 & Rel.-Key Rec. & $2^{51.2} \mathrm{CP}$ & $2^{120.41} \mathrm{HE}$ & not specified & 18 \\
\hline & 28 & 128 & Rel.-Key Imp. & $2^{60} \mathrm{CP}$ & $2^{125.54} \mathrm{HE}$ & not specified & 18 \\
\hline & 31 & 128 & Rel.-Key Imp. & $2^{64} \mathrm{CP}$ & $2^{127.28} \mathrm{HE}$ & $2^{117}$ bytes & 44.2 \\
\hline
\end{tabular}

The security of Hight is investigated in 20 by showing resistance against differential, linear, truncated differential, boomerang, rectangle, impossible differential attacks and their related-key variants. In [2], the safety margin was shown to be 13 rounds as the best attack covers 19 rounds. Recent serious attacks $[19$ by $\mathrm{Lu}$ on reduced round Hight make use of 25, 26 and 28 round impossible differential, related-key rectangle and related-key impossible differential attacks: the last attack is the best attack on HiGHT so far that reduced the safety margin from 13 rounds to four rounds.

In this work, we present the first related-key cryptanalysis of PRESENT. For 128-bit keyed version, we introduce 17-round related-key rectangle attack 2012122 which is not explicitly mentioned in the original proposal 44. Moreover, we further analyze the resistance of HiGHT against impossible differential attacks 2324. Firstly, we improve 25-round impossible differential attack of $\mathrm{Lu}$ by introducing a new characteristic to 26 rounds and update 28 -round relatedkey impossible differential attack on 31 rounds. To the best of our knowledge, these are the best cryptanalytic results on Hight. We provide a summary of our results in Table 1

The organization of the paper is as follows. In Section 2 we give a brief description of the block ciphers PRESENT and Hight. Section 3 introduces the idea behind the related-key attacks on PRESENT and contains related-key rectangle attack on 17-round. In Section 4, we introduce our improved impossible differential and related-key impossible differential attacks on reduced round HiGHT. We conclude with Section 5 and provide supplementary details about the paper in Appendices.

\section{The Block Ciphers PRESENT and HIGHT}

\subsection{Notation}

For Present and Hight, we use the same notation to denote the variables used in this paper. For the sake of clarity and the parallelism with the previous work 
[19, we use exactly the same notation for Hight which is provided in Table 2 Throughout the paper, it is assumed that the rounds are numbered from zero and the leftmost bit is the most significant bit in a byte or a word.

Table 2. Notation

\begin{tabular}{|c|c|}
\hline$\oplus$ & Bitwise logical exclusive OR (XOR) \\
\hline$\boxplus$ & Addition modulo $2^{8}$ \\
\hline$\lll \mathrm{i}$ & Left cyclic rotation by i bits \\
\hline PRESENT- $n-r$ & PRESENT reduced to $r$-rounds with $n$-bit secret key \\
\hline$K_{i}$ & $i$ th subkey of PRESENT \\
\hline$S_{i}$ & $i$ th S-Box of PRESENT \\
\hline$e_{j_{1}, \ldots, j_{k}}$ & A word with zeros in all positions but bits $j_{1}, \ldots, j_{k}$ \\
\hline HIGHT- $r$ & HIGHT reduced to $r$-rounds \\
\hline$e_{j}$ & A byte with zeros in all positions but bit $\mathrm{j}(0 \leqslant j \leqslant 7)$ \\
\hline$e_{j, \sim}$ & $\begin{array}{l}\text { A byte that has zeros in bits } 0 \text { to } j-1 \text {, a one in bit } j \text { and indeterminate } \\
\text { values in bits }(j+1) \text { to } 7\end{array}$ \\
\hline$e_{\bar{j}, \sim}$ & $\begin{array}{l}\text { A byte that has zeros in bits } 0 \text { to } j \text { and indeterminate values in bits }(j+1) \\
\text { to } 7\end{array}$ \\
\hline$?$ & An arbitrary byte \\
\hline$X_{i, j}$ & $j$ th byte of state variable of round $i$ of Hight, $(0 \leqslant j \leqslant 7)(0 \leqslant i \leqslant 32)$ \\
\hline$M K_{i}$ & $i$ th secret key byte of HIGHT \\
\hline$W K_{i}$ & $i$ th whitening key byte of HigHT \\
\hline$S K_{i}$ & $i$ th subkey byte of HiGHT \\
\hline
\end{tabular}

\subsection{PRESENT}

Present is a 31-round (and an output whitening at the end) SPN (Substitution Permutation Network) type block cipher with block size of 64 bits that supports 80 and 128-bit secret key. Round function of PRESENT, which is depicted in Figure 1 is same for both versions of PRESENT and consists of standard operations such as subkey XOR, substitution and permutation: At the beginning of each round, 64-bit input of the round function is XORed with the subkey. Just after the subkey XOR, 16 identical $4 \times 4$-bit S-boxes are used in parallel as a non-linear substitution layer and finally a permutation is performed so as to provide diffusion.

$\mathrm{K}_{\mathrm{i}}$

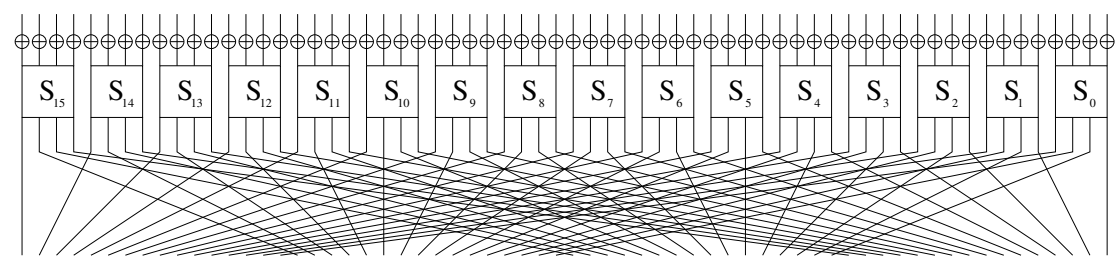

Fig. 1. Round function of Present

The subkeys for each round are derived from the user-provided secret key by the key scheduling algorithm. We provide only the details of the key scheduling algorithm of PRESENT-128 as it is the main target of this paper: 128-bit secret key is stored in a key register $K$ and represented as $k_{127} k_{126} \ldots k_{0}$. The subkeys 
$K_{i}(0 \leq i \leq 31)$ consist of 64 leftmost bits of the actual content of register $K$. After round key $K_{i}$ is extracted, the key register $K$ is rotated by 61 bit positions to the left, then S-box is applied to the left-most eight bits of the key register and finally the round counter value, which is a different constant for each round, is XORed with bits $k_{66} k_{65} k_{64} k_{63} k_{62}$. Further details about the specification of PRESENT are provided in [4].

\section{$2.3 \quad$ HIGHT}

HighT is a 32-round block cipher with 64 -bit block size and 128-bit user key that makes use of an unbalanced Feistel Network. The encryption function starts with an Initial Transformation (IT) that is applied to plaintexts together with input whitening keys $W K \mathrm{~s}$. At the end of 32 rounds, in order to obtain the ciphertexts, a Final Transformation (FT) is applied to the output of the last round together with an output whitening. The byte-oriented round function, shown in Figure 2, uses modular addition, XOR and linear subround functions $F_{0}$ and $F_{1}$; the latter can be described as follows:

$$
\begin{aligned}
& F_{0}(x)=(x \lll 1) \quad \oplus(x \lll 2) \quad \oplus \quad(x \lll 7) \\
& F_{1}(x)=(x \lll 3) \quad \oplus(x \lll 4) \quad \oplus \quad(x \lll 6)
\end{aligned}
$$

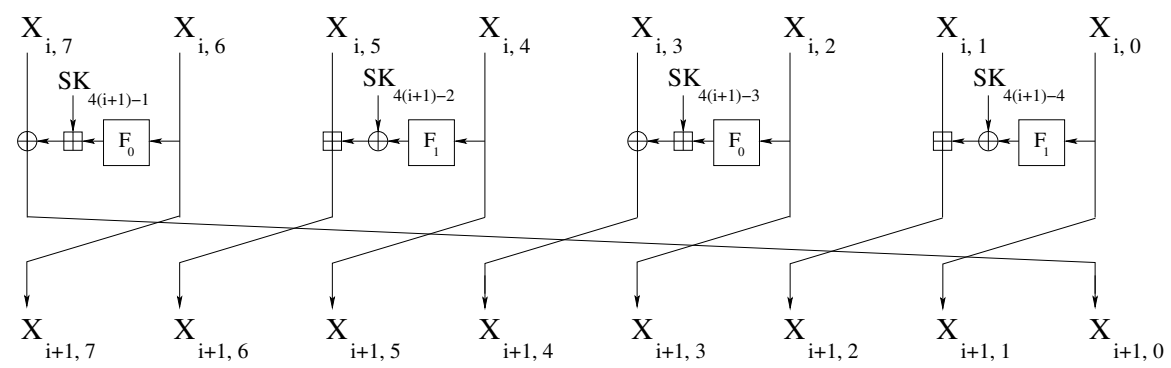

Fig. 2. $i$ th round of Hight for $i=0, \ldots, 31$

HIGHT only works with 128 -bit secret key $M K$ which is treated as 16 bytes, $\left(M K_{15}, \ldots, M K_{0}\right)$. The key schedule of Hight uses additional constants to avoid the self similarity in the key scheduling algorithm which prevents cipher from slide attacks. Input-output whitening keys and round subkeys are obtained by permuting the 16 bytes of the original key and using addition with constants. Table 9 will be extensively used in this paper that shows the relations between the original and the subkey bytes. Namely, each value in a row represents the 
obtained whitening and subkey bytes once the corresponding byte in the first column of the same row of the original key is known. Further details about the specification of Hight are provided in [2]3.

\section{The Related-Key Attacks on PRESENT}

The idea behind the related-key attacks on PRESENT is to benefit from the slow mixing in the key scheduling algorithm which makes use of only one or two Sbox operations (depending on the version) during each iteration. To achieve this goal, we made an efficient search for related-key differentials of PRESENT which was done by flipping at most two bits of the original key. The crucial part of the key differentials is that we only consider the trivial differentials. More precisely, all reduced round key differentials in our attacks work with probability one.

In the original proposal of PRESENT [4, the resistance against differential and linear attacks are given by the bounds provided by the minimum number of active S-boxes. This approach also works for showing resistance against wide variety of attacks. A recent differential attack [12] uses same idea to attack the cipher by increasing the overall probability of the characteristics more effectively. Although there is no contradiction with the security claims given in 4], the differential attack in 12 provides a practical evidence. In this work, however, our aim is quite different and simple in that we try to decrease the number of active S-boxes (NAS). In order to do so, we cancel the intermediate differences with the subkey differences and construct our differentials by activating at most five S-boxes at the beginning. At the end, we are able to construct related-key differentials having less active S-boxes than given in 12. As an example, for PRESEnT-80, the minimum number of active S-boxes for any five-round differential characteristic is given to be ten in [412. However, we found several five-round related-key differentials with only three active S-boxes.

Although it seems quite promising, as the number of rounds increases, the minimum number of active S-boxes gets closer to the one given in the original proposal [12] and the overall probabilities of the characteristics are not optimal. Still, for less number of rounds the related-key differentials are efficient and the number of possible characteristics are quite high. So, attacks like the related-key rectangle attack are easily applicable.

\subsection{The Related-Key Rectangle Attack on PRESENT-128-17}

The related-key rectangle attack is the clever extension of differential cryptanalysis. In rectangle-boomerang style attacks, the attacker uses two short differential characteristics instead of one long differential characteristic. The aim is to benefit from the slow mixing in relatively reduced round versions of the attacked cipher. We provide a brief description about the related-key rectangle attack in Appendix A and follow the mounted attack on Present. Throughout the paper, the related-key rectangle attack is assumed to be mounted by using four related keys. 
Let $E$ denote the encryption function of PRESENT-n- $r$. We treat $E$ as a cascade of four subciphers as $E=E_{f} \circ E_{1} \circ E_{0} \circ E_{b}$ where $E$ is composed of a core $E^{\prime}=E_{1} \circ E_{0}$ covered by additional rounds, $E_{b}$ and $E_{f}$ which are the subciphers before and after the core function respectively.

For the related-key rectangle attack on PRESENT-128-17, we use the following decomposition: $E_{0}$ starts with the first round and ends just after the subkey XOR in round eight. $E_{1}$, on the other hand, commences with the substitution layer in round eight and stops at the end of round 147 Round 0 and round $15-16$ serve as the round before and after the distinguisher respectively $\left(E_{b} \text { and } E_{f}\right)^{8}$

All the differentials used in $E_{0}$ have the same input difference $\alpha=e_{0,1,3,4,5,7}$ and they all work with the key difference $\Delta K^{12}=e_{118,119}$. There are at least 343 such characteristics with varying differences at the beginning of the seventh round: there exist one characteristics of probability $p=2^{-19}, 18$ characteristics of probability $p=2^{-20}, 108$ characteristics of probability $p=2^{-21}$ and 216 characteristics of probability $p=2^{-22}$. Therefore the overall probability for $E_{0}$ is $\hat{p}=\sqrt{1 \cdot\left(2^{-19}\right)^{2}+18 \cdot\left(2^{-20}\right)^{2}+108 \cdot\left(2^{-21}\right)^{2}+216 \cdot\left(2^{-22}\right)^{2}} \approx 2^{-17}$. Table 3 shows one of the characteristics used for $E_{0}$.

Table 3. An example of related-key differential used in $E_{0}$

\begin{tabular}{|c|c|c|c|c|c|c|}
\hline$r$ & $\begin{array}{c}\text { Input } \\
\Delta(I)\end{array}$ & $\begin{array}{c}\text { Key } \\
\Delta(K)\end{array}$ & $\Delta(I) \oplus \Delta(K)$ & $\begin{array}{c}\text { Output } \\
\text { Difference } \\
\Delta(O)\end{array}$ & NAS & $\mathrm{P}$ \\
\hline 1 & $00000000000000 b b$ & 0000000000000000 & $00000000000000 b b$ & 0003000000000000 & 2 & $2^{-4}$ \\
\hline 2 & 0003000000000000 & 0003000000000000 & 0000000000000000 & 0000000000000000 & 0 & 1 \\
\hline 3 & 0000000000000000 & 0000000000000000 & 0000000000000000 & 0000000000000000 & 0 & 1 \\
\hline 4 & 0000000000000000 & $00000 c 0000000000$ & $00000 c 0000000000$ & 0000000004000000 & 1 & $2^{-3}$ \\
\hline 5 & 0000000004000000 & 0000000000000000 & 0000000004000000 & 0000004000000040 & 1 & $2^{-2}$ \\
\hline 6 & 0000004000000040 & 0000003000000000 & 0000007000000040 & 0000000200000202 & 2 & $2^{-4}$ \\
\hline 7 & 0000000200000202 & 0000000000000000 & 0000000200000202 & 0000010500000105 & 3 & $2^{-6}$ \\
\hline 8 & 0000010500000105 & $00000000 c 0000000$ & $00000105 c 0000105$ & & & 1 \\
\hline
\end{tabular}

Given the $\alpha$ difference, the number of active S-boxes in $E_{b}$ which lead to an $\alpha$ difference in round 1 can be found by applying the inverse permutation to $\alpha$ difference. This leads to six active S-boxes in the first round with varying output differences after the substitution layer. These six S-boxes are used to create the $\alpha$ difference before the core function. Since the output difference is known for all active S-boxes in round 0 , namely $1_{x}$, not all of the input differences are possible. The number of possible input differences is only $2^{15.5}$ instead of $2^{24}$.

For the second subcipher $E_{1}$, we use the fixed output difference $\delta=e_{11,15}$ under the key difference $\Delta K^{13}=e_{117,121}$. The most efficient characteristic is provided in Table 4 with probability $p=2^{-12}$. As it can be seen from the table, the overall probability for $E_{1}$ is $\hat{q} \approx 2^{-12}$. Thus, the probability of the relatedkey rectangle distinguisher is given by $\operatorname{Pr}=2^{-64} \hat{p}^{2} \hat{q}^{2} \approx 2^{-122}$. Similarly, the subcipher $E_{f}$ after the core function can be defined by letting $\delta$ propagate. In

\footnotetext{
7 This decomposition is not unique and can be done in various ways.

${ }^{8}$ We exclude the output whitening in our attack
} 
round 15 , there exist two active S-boxes with input differences $8_{x}$ each leading to six output differences and these outputs are diffused to six different S-boxes after key addition in round 16 which produce $2^{21.22}$ possible output differences in total out of $2^{24}$.

Table 4. An example of related-key differential used in $E_{1}$

\begin{tabular}{|c|c|c|c|c|c|c|}
\hline$r$ & $\begin{array}{c}\text { Output } \\
\text { Difference } \\
\Delta(O)\end{array}$ & $\begin{array}{c}\text { Key } \\
\text { Difference }\end{array}$ & $\Delta(I) \oplus \Delta(K)$ & $P^{-1}\left(S^{-1}(I \oplus K)\right)$ & NAS & P \\
\hline 14 & 0000000000008800 & 0000000000008800 & 0000000000000000 & 0000000000000000 & 0 & 1 \\
\hline 13 & 0000000000000000 & 0000000000000000 & 0000000000000000 & 0000000000000000 & 0 & 1 \\
\hline 12 & 0000000000000000 & 0000000000220000 & 0000000000220000 & 0000000000600060 & 2 & $2^{-6}$ \\
\hline 11 & 0000000000600060 & 0000000000000000 & 0000000000600060 & 0000000008800000 & 2 & $2^{-6}$ \\
\hline 10 & 0000000008800000 & 0000000008800000 & 0000000000000000 & 0000000000000000 & 0 & 1 \\
\hline 9 & 0000000000000000 & 0000000000000000 & 0000000000000000 & 0000000000000000 & 0 & 1 \\
\hline 8 & & & 0000000000000000 & 0000000000000000 & & \\
\hline
\end{tabular}

To attack 17 round PRESENT, we request $2^{39}$ structures of $2^{24}$ plaintexts each. The structures are chosen in such a way that each structure varies all over the possible inputs to the active S-boxes in $E_{b}$, while the differences for the other S-boxes are kept zero. Our aim is to get an $\alpha$ difference at the beginning of $E_{0}$. This technique for choosing plaintexts lets us $2^{47}$ pairs in total for each structure in which $2^{23}$ of them satisfy $\alpha$ difference before $E_{0}$. Thus, the total number of pairs with an $\alpha$ difference before the core function is $2^{62}$ that produce approximately $2^{124}$ quartets of which $2^{124} \cdot 2^{-64} \cdot 2^{-58}=2^{2}=4$ are expected to be right. The overall attack works as follows:

1. Generate $2^{39}$ structures of $2^{24}$ plaintexts and encrypt each structure of plaintexts with $K^{1}, K^{2}, K^{3}$ and $K^{4}$ to obtain the corresponding pool of ciphertexts $C^{j}$ where $1 \leq j \leq 4$.

- This step requires data complexity of $2^{63}$ chosen plaintexts and time complexity of $2^{65}$ PRESENT-128-17 encryptions.

2. Generate $2^{24+8+24}=2^{56}$ counters each of which corresponds to a different key guess in $E_{b}$ and $E_{f}$ respectively.

- Time complexity of this step is $2^{56}$ memory accesses.

3. Insert $2^{65}$ ciphertexts $\left(C^{1}, C^{3}\right)$ and $\left(C^{2}, C^{4}\right)$ into hash tables $\left(T_{13}^{1}, T_{13}^{3}\right)$ and $\left(T_{24}^{2}, T_{24}^{4}\right)$ respectively indexed by 40 (expected inactive) bits. If a collision occurs in the same bins of $\left(T_{13}^{1}, T_{13}^{3}\right)$ and $\left(T_{24}^{2}, T_{24}^{4}\right)$, check whether the differences of the collided ciphertexts are one of the $2^{21.22}$ expected ciphertext differences.

- This step has time complexity of $2^{65}$ memory accesses from inserting all the ciphertext into hash tables. In the hash tables there exist $2^{40}$ bins and in each bin we expect to have $2^{23}$ ciphertexts. Therefore, we can form $\left(2^{23}\right)^{2}=2^{46}$ pairs where one of the components from $T_{13}^{1}\left(T_{24}^{2}\right)$ and the other is from $T_{13}^{3}\left(T_{24}^{4}\right)$. That makes $2^{86}$ pairs in total for each pair of tables $\left(T_{13}^{1}, T_{13}^{3}\right)$ and $\left(T_{24}^{2}, T_{24}^{4}\right)$. In order to check whether colliding 
ciphertexts' differences are one of the expected ciphertext differences we have to make $2^{87}$ memory accesses in total. The number of remaining pairs is $2^{86-2.78}=2^{83.22}$ for each of $\left(T_{13}^{1}, T_{13}^{3}\right)$ and $\left(T_{24}^{2}, T_{24}^{4}\right)$ since the probability of having expected difference in the ciphertexts is $2^{21.22-24}=$ $2^{-2.78}$.

4. For each surviving pair $\left(C_{1}, C_{3}\right)$ (and $\left.\left(C_{2}, C_{4}\right)\right)$ from the previous step, find the corresponding plaintext pairs $\left(P_{1}, P_{3}\right)$ (and $\left.\left(P_{2}, P_{4}\right)\right)$ from the structures. For each such pair, check whether $P_{1} \oplus P_{2}$ satisfies the required difference in $E_{b}$. If this check succeeds, examine the ciphertexts $C_{3}$ and $C_{4}$ that collided with $C_{1}$ and $C_{2}$ respectively. If the difference between the corresponding plaintexts $P_{3}$ and $P_{4}$ also satisfies the required difference in $E_{b}$, continue to analyze the quartet $\left(\left(P_{1}, P_{2}\right),\left(P_{3}, P_{4}\right)\right)$.

- The probability that $P_{1} \oplus P_{2}$ satisfies the required difference is $2^{15.5-24}=$ $2^{-8.5}$, if they are in the same structure. So, the probability that the required difference is satisfied is $2^{-8.5-39}=2^{-47.5}$ under the assumption of uniform distribution of plaintexts and structures. This reduces the number of pairs satisfying the condition in $\left(T_{13}^{1}, T_{13}^{3}\right)$ to $2^{83.22-47.5}=$ $2^{35.72}$. Similarly, there exist $2^{35.72}$ pairs in $\left(T_{24}^{2}, T_{24}^{4}\right)$. Thus, we can form $\left(2^{35.72}\right)^{2}=2^{71.44}$ quartets satisfying the conditions in $E_{b}$ and $E_{f}$. In order to do this filtering we have to make $2^{84.22}$ memory accesses.

5 . For each remaining quartet $\left(\left(P_{1}, P_{2}\right),\left(P_{3}, P_{4}\right)\right),\left(\left(C_{1}, C_{2}\right),\left(C_{3}, C_{4}\right)\right)$ and every possible subkey value ( $k_{b}$ and $k_{f}$ independently) of $E_{b}$ and $E_{f}$ test whether

$$
\begin{array}{ll}
E_{b_{k_{b}}}\left(P_{1}\right) \oplus E_{k_{b}^{\prime}}\left(P_{2}\right)=E_{k_{b}^{\prime \prime}}\left(P_{3}\right) \oplus E_{k_{b}^{\prime \prime \prime}}\left(P_{4}\right)=\alpha & \text { where } k_{b}^{\prime}=k_{b} \oplus \Delta K^{12} \\
E_{f_{k_{f}}}^{-1}\left(C_{1}\right) \oplus E_{f_{k_{f}^{\prime \prime}}^{-1}}^{-1}\left(C_{3}\right)=E_{f_{k_{f}^{\prime}}}^{-1}\left(C_{2}\right) \oplus E_{f_{k_{f}^{\prime \prime \prime}}}^{-1}\left(P_{4}\right)=\delta & \text { and } k_{b}^{\prime \prime}=k_{b}^{\prime \prime \prime} \oplus \Delta K^{12}, \\
& \text { where } k_{f}^{\prime \prime}=k_{f} \oplus \Delta K^{13} \\
& \text { and } k_{f}^{\prime}=k_{f}^{\prime \prime \prime} \oplus \Delta K^{13} \text { hold. }
\end{array}
$$

If this is the case, increment the counters that correspond to $k_{b}, k_{f}$.

- In this step, every surviving quartet is partially encrypted (in $E_{b}$ ) and decrypted (in $E_{f}$ ) independently. As the number of subkeys guessed are more in $E_{f}$ than in $E_{b}$, the overall complexity of this step is $2^{71.44+32}=$ $2^{103.44}$ memory accesses and half round decryptions (as eight $\mathrm{S}$ boxes are affected in total); the latter is equivalent to $2^{99}$ PRESENT-128-17 evaluations.

6. Output the subkeys whose counters are maximal.

- This step requires $2^{56}$ memory accesses.

Since $\alpha$ difference after $E_{b}$ can be obtained from $2^{15.5}$ input differences in step 5 , the probability that the intermediate difference is $\alpha$ before the core function is $2^{-15.5}$ on average. Thus, each subkey is suggested by a quartet with probability $2^{-31}$. Similarly, the probability that the difference after the core function has an $\delta$ difference is $2^{-21.22}$ on average leading to $2^{-42.44}$ of the subkeys by the quartets. 
Each of the $2^{71.44}$ quartets that enter step 5 suggests $2^{56-2 \times 15.5-2 \times 21.22}=2^{-17.44}$ subkeys, so the total number of suggested subkeys is about $2^{54}$. As there are $2^{56}$ subkeys, the expected number of times a wrong subkey is suggested is about $2^{-2}$. This means that we can find the right subkey or at least discard almost all the wrong subkeys.

Thus, the overall attack has memory complexity of $2^{53}$ bytes, time complexity of $2^{104}$ memory accesses and data complexity of $2^{63}$ chosen plaintexts. The expected number of right quartets is taken to be four.

\section{Impossible Differential Attacks on HIGHT}

In this section, we introduce improved impossible differential attack on 26-round and related-key impossible differential attack on 31-round HiGHT which utilize 16-round impossible differential and 22-round related-key impossible differential characteristics respectively. For impossible differential attack on 26-round Hight, we use a similar characteristic as in [19] which enables us to attack 26round of HIGHT with a lower complexity. However, we use better characteristic for related-key impossible differential attack.

The process of both attacks is similar. First, a data collection part is processed for the generation of necessary plaintext-ciphertext pairs. Then, to guarantee the impossible differential characteristic, pairs are filtered by checking the conditions at each intermediate rounds. At the end, the guessed key is eliminated if any one of the remaining pairs satisfies the impossible differential characteristic.

\subsection{Impossible Differential Attack on HIGHT-26}

We use the following 16-round impossible differential which is also given in Table 5 in detail:

$$
\left(e_{0, \sim}, 0,0,0,0,0,0,0\right) \nrightarrow\left(0,80_{x}, 0,0,0,0,0,0\right)
$$

In Table 5 the contradictory differences and the guessed subkey bytes in the attack are labeled with gray background. The differences used here are considered with respect to XOR operation and shown as hexadecimal. The propagation of the differences can easily be checked by the properties of addition and linear subround functions $F_{i}$. Contradiction is shown by miss in the middle manner at values $X_{13,7}$. This attack covers the rounds $0-25$ and excludes the input whitening as done in [19]. Overall attack on 26-round HiGHT works as follows:

\section{1.) Data Collection}

(i) Choose $2^{13}$ structures of $2^{48}$ plaintexts each where the bytes $(1,0)$ have fixed values, bytes $(7,6,5,4,3)$ and most significant 7 bits of the byte $(2)$ take all possible values. 
Table 5. 26-Round impossible differential

\begin{tabular}{|c|c|c|c|c|c|c|c|c|c|c|c|c|}
\hline & $\Delta X_{i, 7}$ & $\Delta X_{i, 6}$ & $\Delta X_{i, 5}$ & $\Delta X_{i, 4}$ & $\Delta X_{i, 3}$ & $\Delta X_{i, 2}$ & $\Delta X_{i, 1}$ & $\Delta X_{i, 0}$ & \multicolumn{4}{|c|}{ Subkeys } \\
\hline$\Delta X_{0}$ & $?$ & $?$ & $?$ & $?$ & $?$ & $e_{0, \sim}$ & 0 & 0 & $S K_{3}$ & $S K_{2}$ & $S K_{1}$ & $S K_{0}$ \\
\hline$\Delta X_{1}$ & $?$ & $?$ & $?$ & $?$ & $e_{0, \sim}$ & 0 & 0 & 0 & $S K_{7}$ & $S K_{6}$ & $S K_{5}$ & $S K_{4}$ \\
\hline$\Delta X_{2}$ & $?$ & $?$ & $?$ & $e_{0, \sim}$ & 0 & 0 & 0 & 0 & $S K_{11}$ & $S K_{10}$ & $S K_{9}$ & $S K_{8}$ \\
\hline$\Delta X_{3}$ & $?$ & $?$ & $e_{0, \sim}$ & 0 & 0 & 0 & 0 & 0 & $S K_{15}$ & $S K_{14}$ & $S K_{13}$ & $S K_{12}$ \\
\hline$\Delta X_{4}$ & $?$ & $e_{0, \sim}$ & 0 & 0 & 0 & 0 & 0 & 0 & $S K_{19}$ & $S K_{18}$ & $S K_{17}$ & $S K_{16}$ \\
\hline$\Delta X_{5}$ & $e_{0, \sim}$ & 0 & 0 & 0 & 0 & 0 & 0 & 0 & $S K_{23}$ & $S K_{22}$ & $S K_{21}$ & $S K_{20}$ \\
\hline$\Delta X_{6}$ & 0 & 0 & 0 & 0 & 0 & 0 & 0 & $e_{0, \sim}$ & $S K_{27}$ & $S K_{26}$ & $S K_{25}$ & $S K_{24}$ \\
\hline$\Delta X_{7}$ & 0 & 0 & 0 & 0 & 0 & $?$ & $e_{0, \sim}$ & 0 & $S K_{31}$ & $S K_{30}$ & $S K_{29}$ & $S K_{28}$ \\
\hline$\Delta X_{8}$ & 0 & 0 & 0 & $?$ & $?$ & $e_{0, \sim}$ & 0 & 0 & $S K_{35}$ & $S K_{34}$ & $S K_{33}$ & $S K_{32}$ \\
\hline$\Delta X_{9}$ & 0 & $?$ & $?$ & $?$ & $e_{0, \sim}$ & 0 & 0 & 0 & $S K_{39}$ & $S K_{38}$ & $S K_{37}$ & $S K_{36}$ \\
\hline$\Delta X_{10}$ & ? & ? & $?$ & $e_{0, \sim}$ & 0 & 0 & 0 & $?$ & $S K_{43}$ & $S K_{42}$ & $S K_{41}$ & $S K_{40}$ \\
\hline$\Delta X_{11}$ & $?$ & $?$ & $e_{0, \sim}$ & 0 & 0 & $?$ & $?$ & $?$ & $S K_{47}$ & $S K_{46}$ & $S K_{45}$ & $S K_{44}$ \\
\hline$\Delta X_{12}$ & $?$ & $e_{0, \sim}$ & 0 & $?$ & $?$ & $?$ & $?$ & $?$ & $S K_{51}$ & $S K_{50}$ & $S K_{49}$ & $S K_{48}$ \\
\hline$\Delta X_{13}$ & $e_{0, \sim}$ & $?$ & $?$ & $?$ & $?$ & $?$ & $?$ & $?$ & $S K_{55}$ & $S K_{54}$ & $S K_{53}$ & $S K_{52}$ \\
\hline$\Delta X_{13}$ & $e_{\overline{0}}, \sim$ & $80_{x}$ & $?$ & $?$ & $?$ & $?$ & $?$ & $?$ & $S K_{55}$ & $S K_{54}$ & $S K_{53}$ & $S K_{52}$ \\
\hline$\Delta X_{14}$ & $80_{x}$ & 0 & $?$ & $?$ & $?$ & $?$ & $?$ & $e_{0, \sim}$ & $S K_{59}$ & $S K_{58}$ & $S K_{57}$ & $S K_{56}$ \\
\hline$\Delta X_{15}$ & 0 & 0 & $?$ & $?$ & $?$ & $?$ & $e_{0, \sim}$ & $80_{x}$ & $S K_{63}$ & $S K_{62}$ & $S K_{61}$ & $S K_{60}$ \\
\hline$\Delta X_{16}$ & 0 & 0 & $?$ & $?$ & $?$ & $e_{0, \sim}$ & $80_{x}$ & 0 & $S K_{67}$ & $S K_{66}$ & $S K_{65}$ & $S K_{64}$ \\
\hline$\Delta X_{17}$ & 0 & 0 & $?$ & $?$ & $e_{0, \sim}$ & $80_{x}$ & 0 & 0 & $S K_{71}$ & $S K_{70}$ & $S K_{69}$ & $S K_{68}$ \\
\hline$\Delta X_{18}$ & 0 & 0 & $?$ & $e_{2, \sim}$ & $80_{x}$ & 0 & 0 & 0 & $S K_{75}$ & $S K_{74}$ & $S K_{73}$ & $S K_{72}$ \\
\hline$\Delta X_{19}$ & 0 & 0 & $e_{2, \sim}$ & $80_{x}$ & 0 & 0 & 0 & 0 & $S K_{79}$ & $S K_{78}$ & $S K_{77}$ & $S K_{76}$ \\
\hline$\Delta X_{20}$ & 0 & 0 & $80_{x}$ & 0 & 0 & 0 & 0 & 0 & $S K_{83}$ & $S K_{82}$ & $S K_{81}$ & $S K_{80}$ \\
\hline$\Delta X_{21}$ & 0 & $80_{x}$ & 0 & 0 & 0 & 0 & 0 & 0 & $S K_{87}$ & $S K_{86}$ & $S K_{85}$ & $S K_{84}$ \\
\hline$\Delta X_{22}$ & $80 x$ & 0 & 0 & 0 & 0 & 0 & 0 & $e_{0, \sim}$ & $S K_{91}$ & $S K_{90}$ & $S K_{89}$ & $S K_{88}$ \\
\hline$\Delta X_{23}$ & 0 & 0 & 0 & 0 & 0 & $?$ & $e_{0, \sim}$ & $80_{x}$ & $S K_{95}$ & $S K_{94}$ & $S K_{93}$ & $S K_{92}$ \\
\hline$\Delta X_{24}$ & 0 & 0 & 0 & $?$ & $?$ & $e_{0, \sim}$ & $80_{x}$ & 0 & $S K_{99}$ & $S K_{98}$ & $S K_{97}$ & $S K_{96}$ \\
\hline$\Delta X_{25}$ & 0 & $?$ & $?$ & $?$ & $e_{0, \sim}$ & $80_{x}$ & 0 & 0 & $S K_{103}$ & $S K_{102}$ & $S K_{101}$ & $S K_{100}$ \\
\hline$\Delta X_{26}$ & $?$ & $?$ & $?$ & $e_{\overline{0}}, \sim$ & $80_{x}$ & 0 & 0 & $?$ & $W K_{7}$ & $W K_{6}$ & $W K_{5}$ & $W K_{4}$ \\
\hline$F T$ & $?$ & $?$ & $?$ & $?$ & $e_{\overline{0}, \sim}$ & $80_{x}$ & 0 & 0 & & & & \\
\hline
\end{tabular}

- Such a structure of plaintexts propose $2^{94}$ plaintext pairs and so we get $2^{107}$ pairs in total.

(ii) Obtain all the ciphertexts $C^{i}$ of the plaintexts $P^{i}$. Choose only the ciphertext pairs satisfying the difference $\left(?, ?, ?, ?, e_{\overline{0}, \sim}, 80_{x}, 0,0\right)$.

- This step can be done by inserting all the ciphertexts into a hash table indexed by expected inactive bits and choosing the colliding pairs which satisfy the required difference. There is a 25 -bit filtering condition over the ciphertext pairs. Therefore, $2^{82}$ pairs remain.

\section{2.) Filtering and Key Elimination}

We have 28 similar steps given in Table 6 to reach impossible differential characteristic and eliminate the wrong key values.

Let us look at the first step as an example. Guess $M K_{3}$ and partially encrypt every plaintext pairs by using $S K_{3}$ to obtain $(7,0)$-th bytes of $X_{1}$ (The relation between the $M K$ values and $S K$ values are given in the Table 9). The expected difference for the $(7,0)$-th bytes is $(?, 0)$ which comes up with an eight-bit condition. Therefore, the number of total pairs is decreased to $2^{82-8}=2^{74}$ after this step. In this step, we partially encrypt $2^{82}$ pairs with the guessed eight-bit of the secret key. Each partial encryption is equivalent to 1/4th of a round of HiGHT and the overall attack is done on 26 rounds. Thus, the complexity of this step is $2 \cdot 2^{82} \cdot 2^{8} \cdot \frac{1}{4} \cdot \frac{1}{26} \approx 2^{84.30} 26$-round HigHT encryptions. Remaining steps given in Table 6 follow similarly. Moreover, if the secret key byte $M K$ is already guessed 
and known for the required subkey $S K$, it is directly used and since most of the previous conditions are preserved for the next rounds there does not exist too much conditions on the evaluation process of intermediate rounds.

Table 6. 26-Round impossible differential attack

\begin{tabular}{|c|c|c|c|c|c|c|c|}
\hline & $\begin{array}{c}\text { Guess } \\
\text { Key Byte }\end{array}$ & Use & Obtain & \begin{tabular}{c|} 
Check \\
Difference
\end{tabular} & $\begin{array}{c}\text { Condition } \\
\text { (In terms of bits) }\end{array}$ & $\begin{array}{c}\text { Remaining } \\
\text { Pairs }\end{array}$ & $\begin{array}{c}\text { Time } \\
\text { Complexity(HE) }\end{array}$ \\
\hline 1 & $M K_{3}$ & $S K_{3}$ & $(7,0)$ of $X_{1}$ & $(?, 0)$ & 8 & $2^{74}$ & $\approx 2^{84.30}$ \\
\hline 2 & $M K_{1}$ & $W K_{7}, S K_{103}$ & $(7,6)$ of $X_{25}$ & $(0, ?)$ & 8 & $2^{66}$ & $\approx 2^{84.30}$ \\
\hline 3 & $M K_{2}$ & $S K_{2}$ & $(6,5)$ of $X_{1}$ & - & - & $2^{66}$ & $\approx 2^{84.30}$ \\
\hline 4 & $M K_{7}$ & $S K_{7}$ & $(7,0)$ of $X_{2}$ & $(?, 0)$ & 8 & $2^{58}$ & $\approx 2^{92.30}$ \\
\hline 5 & $M K_{0}$ & $W K_{6}, S K_{102}$ & $(5,4)$ of $X_{25}$ & - & - & $2^{58}$ & $\approx 2^{92.30}$ \\
\hline 6 & $M K_{4}$ & $S K_{98}$ & $(5,4)$ of $X_{24}$ & $(0, ?)$ & 8 & $2^{50}$ & $\approx 2^{100.30}$ \\
\hline 7 & - & $W K_{5}, S K_{101}$ & $(3,2)$ of $X_{25}$ & - & - & $2^{50}$ & $\approx 2^{92.30}$ \\
\hline 8 & - & $S K_{97}$ & $(3,2)$ of $X_{24}$ & - & - & $2^{50}$ & $\approx 2^{92.30}$ \\
\hline 9 & $M K_{8}$ & $S K_{93}$ & $(3,2)$ of $X_{23}$ & $(0, ?)$ & 8 & $2^{42}$ & $\approx 2^{100.30}$ \\
\hline 10 & - & $S K_{1}$ & $(4,3)$ of $X_{1}$ & - & - & $2^{42}$ & $\approx 2^{92.30}$ \\
\hline 11 & $M K_{6}$ & $S K_{6}$ & $(6,5)$ of $X_{2}$ & - & - & $2^{42}$ & $\approx 2^{100.30}$ \\
\hline 12 & $M K_{11}$ & $S K_{11}$ & $(7,0)$ of $X_{3}$ & $(?, 0)$ & 8 & $2^{34}$ & $\approx 2^{108.30}$ \\
\hline 13 & - & $W K_{4}, S K_{100}$ & $(1,0)$ of $X_{25}$ & - & - & $2^{34}$ & $\approx 2^{100.30}$ \\
\hline 14 & - & $S K_{96}$ & $(1,0)$ of $X_{24}$ & - & - & $2^{34}$ & $\approx 2^{100.30}$ \\
\hline 15 & $M K_{15}$ & $S K_{92}$ & $(1,0)$ of $X_{23}$ & - & - & $2^{34}$ & $\approx 2^{108.30}$ \\
\hline 16 & - & $S K_{88}$ & $(1,0)$ of $X_{22}$ & $\left(0, e_{0, \sim)}\right.$ & 8 & $2^{26}$ & $\approx 2^{108.30}$ \\
\hline 17 & - & $S K_{0}$ & $(2,1)$ of $X_{1}$ & - & - & $2^{26}$ & $\approx 2^{100.30}$ \\
\hline 18 & $M K_{5}$ & $S K_{5}$ & $(4,3)$ of $X_{2}$ & - & - & $2^{26}$ & $\approx 2^{108.30}$ \\
\hline 19 & $M K_{10}$ & $S K_{10}$ & $(6,5)$ of $X_{3}$ & - & - & $2^{26}$ & $\approx 2^{116.30}$ \\
\hline 20 & - & $S K_{15}$ & $(7,0)$ of $X_{4}$ & $(?, 0)$ & 8 & $2^{18}$ & $\approx 2^{116.30}$ \\
\hline 21 & - & $S K_{99}$ & $(7,6)$ of $X_{24}$ & - & - & $2^{18}$ & $\approx 2^{108.30}$ \\
\hline 22 & - & $S K_{95}$ & $(7,6)$ of $X_{23}$ & - & - & $2^{18}$ & $\approx 2^{108.30}$ \\
\hline 23 & $M K_{14}$ & $S K_{91}$ & $(7,6)$ of $X_{22}$ & - & - & $2^{18}$ & $\approx 2^{116.30}$ \\
\hline 24 & - & $S K_{87}$ & $(7,6)$ of $X_{21}$ & $\left(0,80_{x}\right)$ & 7 & $2^{11}$ & $\approx 2^{116.30}$ \\
\hline 25 & - & $S K_{4}$ & $(2,1)$ of $X_{2}$ & - & - & $2^{11}$ & $\approx 2^{109.30}$ \\
\hline 26 & $M K_{9}$ & $S K_{9}$ & $(4,3)$ of $X_{3}$ & - & - & $2^{11}$ & $\approx 2^{117.30}$ \\
\hline 27 & - & $S K_{14}$ & $(6,5)$ of $X_{4}$ & - & - & $2^{11}$ & $\approx 2^{117.30}$ \\
\hline 28 & - & $S K_{19}$ & $(7,0)$ of $X_{5}$ & $\left(e_{0, \sim, 0)}\right.$ & 8 & - & $\approx 2^{114.30}$ \\
\hline
\end{tabular}

In step 28, if a pair satisfies the impossible differential characteristic, we eliminate that guessed key. Since there is an eight-bit condition, every pair eliminates $2^{-8}$ of the keys. Therefore after the first pair, there remain $2^{112}-2^{104}=$ $2^{112} \cdot\left(1-2^{-8}\right)$ keys. After the second pair, it is expected to have $2^{112} \cdot\left(1-2^{-8}\right)-$ $2^{112} \cdot\left(1-2^{-8}\right) \cdot 2^{-8}=2^{112} \cdot\left(1-2^{-8}\right)^{2}$ remaining keys. Following that manner, after the last pair, we have $2^{112}\left(1-2^{-8}\right)^{2^{11}} \approx 2^{100.46}$ remaining keys. Complexity of this step is $2 \cdot 2^{112}\left\{1+\left(1-2^{-8}\right)+\ldots+\left(1-2^{-8}\right)^{2^{11}-1}\right\} \cdot \frac{1}{4} \cdot \frac{1}{26} \approx 2^{114.30}$ HigHT encryptions.

\section{3.) Final Step}

For every recorded 112 bit key at the end of Step 28, we obtain the remaining 16 bits and the original key itself with exhaustive search by checking over two plaintext-ciphertext pairs. The probability that a wrong key is suggested is approximately $2^{-64 \times 2}=2^{-128}$. So, the expected number of wrong keys is about $2^{-128} \cdot 2^{116.46}=2^{-11.54}$. Thus, it is very likely that we can find the correct key.

The total complexity of the steps given in Table 6 is $2^{119.35}$. Since we have approximately $2^{100.46}$ remaining keys before the final step, the complexity of the 
final step is $2^{100.46+16}=2^{116.46}$. Therefore, the overall complexity of the attack is $2^{119.35}+2^{116.46}=2^{119.53} 26$-round HiGHT evaluations, $2^{61}$ chosen plaintexts of data and $2^{109}$ bytes of memory.

\subsection{Related-Key Impossible Differential Attack on HIGHT-31}

In this section, we introduce our related-key impossible differential attack on 31-round HiGHT which utilizes a new 22-round related-key impossible differential. The differences of this attack from 19 are the used related-key impossible differential and the overall complexity which makes use of a related-key impossible differential of three more rounds. We use the following 22-round impossible differential which is given in Table 7 in detail:

$$
\left(0,0,0,0,0,0,80_{x}, 0\right) \nrightarrow\left(0,0,0,80_{x}, 0,0,0,0\right)
$$

Table 7. 31-Round related-key impossible differential

\begin{tabular}{|c|c|c|c|c|c|c|c|c|c|c|c|c|}
\hline & $\Delta X_{i, 7}$ & $\Delta X_{i, 6}$ & $\Delta X_{i, 5}$ & $\Delta X_{i, 4}$ & $\Delta X_{i, 3}$ & $\Delta X_{i, 2}$ & $\Delta X_{i, 1}$ & $\Delta X_{i, 0}$ & \multicolumn{4}{|c|}{ Subkeys } \\
\hline$\Delta X_{0}$ & $?$ & $?$ & & $e_{0, \sim}$ & $80_{x}$ & 0 & $?$ & $?$ & $S K_{3}$ & $S K_{2}$ & $S K_{1}$ & $S K_{0}$ \\
\hline$\Delta X_{1}$ & $?$ & $?$ & $e_{0, \sim}$ & $80_{x}$ & 0 & 0 & $?$ & $?$ & $S K_{7}$ & $S K_{6}$ & $S K_{5}$ & $S K_{4}$ \\
\hline$\Delta X_{2}$ & $?$ & $e_{0, \sim}$ & $80_{x}$ & 0 & 0 & 0 & $?$ & $?$ & $S K_{11}$ & $S K_{10}$ & $S K_{9}$ & $S K_{8}$ \\
\hline$\Delta X_{3}$ & $e_{0, \sim}$ & $80_{x}$ & 0 & 0 & 0 & 0 & ? & ? & $S K_{15}$ & $S K_{14}$ & $S K_{13}$ & $S K_{12}$ \\
\hline$\Delta X_{4}$ & $80_{x}$ & 0 & 0 & 0 & 0 & 0 & $?$ & $e_{2, \sim}$ & $S K_{19}$ & $S K_{18}$ & $S K_{17}$ & $S K_{16}$ \\
\hline$\Delta X_{5}$ & 0 & 0 & 0 & 0 & 0 & 0 & $e_{2, \sim}$ & $80_{x}$ & $S K_{23}$ & $S K_{22}$ & $S K_{21}$ & $S K_{20}$ \\
\hline$\Delta X_{6}$ & 0 & 0 & 0 & 0 & 0 & 0 & $80_{x}$ & 0 & $S K_{27}$ & $S K_{26}$ & $S K_{25}$ & $S K_{24}$ \\
\hline$\Delta X_{7}$ & 0 & 0 & 0 & 0 & 0 & 0 & 0 & 0 & $S K_{31}$ & $S K_{30}$ & $S K_{29}$ & $S K_{28}$ \\
\hline$\Delta X_{8}$ & 0 & 0 & 0 & 0 & 0 & 0 & 0 & 0 & $S K_{35}$ & $S K_{34}$ & $S K_{33}$ & $S K_{32}$ \\
\hline$\Delta X_{9}$ & 0 & 0 & 0 & 0 & 0 & 0 & 0 & 0 & $S K_{39}$ & $S K_{38}$ & $\mathrm{SK}_{37}$ & $S K_{36}$ \\
\hline$\Delta X_{10}$ & 0 & 0 & 0 & 0 & 0 & 0 & 0 & 0 & $\mathrm{SK}_{43}$ & $S K_{42}$ & $\mathrm{SK}_{41}$ & $S K_{40}$ \\
\hline$\Delta X_{11}$ & 0 & 0 & 0 & $80_{x}$ & 0 & 0 & 0 & 0 & $S K_{47}$ & $S K_{46}$ & $S K_{45}$ & $S K_{44}$ \\
\hline$\Delta X_{12}$ & 0 & $e_{2, \sim}$ & $80_{x}$ & 0 & 0 & 0 & 0 & 0 & $S K_{51}$ & $S K_{50}$ & $S K_{49}$ & $S K_{48}$ \\
\hline$\Delta X_{13}$ & $e_{2, \sim}$ & $80_{x}$ & 0 & 0 & 0 & 0 & 0 & $?$ & $S K_{55}$ & $S K_{54}$ & $S K_{53}$ & $S K_{52}$ \\
\hline$\Delta X_{14}$ & $80_{x}$ & 0 & 0 & 0 & 0 & $?$ & $?$ & $e_{0, \sim}$ & $S K_{59}$ & $S K_{58}$ & $S K_{57}$ & $S K_{56}$ \\
\hline$\Delta X_{15}$ & 0 & $80_{x}$ & 0 & $?$ & $?$ & $?$ & $e_{0, \sim}$ & $80_{x}$ & $S K_{63}$ & $S K_{62}$ & $S K_{61}$ & $S K_{60}$ \\
\hline$\Delta X_{16}$ & $80_{x}$ & $?$ & $?$ & $?$ & $?$ & $e_{0, \sim}$ & $80_{x}$ & $e_{0, \sim}$ & $S K_{67}$ & $S K_{66}$ & $S K_{65}$ & $S K_{64}$ \\
\hline$\Delta X_{17}$ & $?$ & $?$ & $?$ & ? & $e_{0, \sim}$ & $?$ & $e_{0, \sim}$ & $?$ & $S K_{71}$ & $S K_{70}$ & $S K_{69}$ & $S K_{68}$ \\
\hline$\Delta X_{17}$ & $?$ & ? & ? & $e_{0, \sim}$ & $80_{x}$ & 0 & $?$ & $?$ & $S K_{71}$ & $S K_{70}$ & $S K_{69}$ & $S K_{68}$ \\
\hline$\Delta X_{18}$ & $?$ & $?$ & $e_{0, \sim}$ & $80_{x}$ & 0 & 0 & $?$ & $?$ & $S K_{75}$ & $S K_{74}$ & $S K_{73}$ & $S K_{72}$ \\
\hline$\Delta X_{19}$ & $?$ & $e_{0, \sim}$ & $80_{x}$ & 0 & 0 & 0 & $?$ & $?$ & $S K_{79}$ & $S K_{78}$ & $S K_{77}$ & $S K_{76}$ \\
\hline$\Delta X_{20}$ & $e_{0, \sim}$ & $80_{x}$ & 0 & 0 & 0 & 0 & $?$ & ? & $S K_{83}$ & $S K_{82}$ & $S K_{81}$ & $S K_{80}$ \\
\hline$\Delta X_{21}$ & $80_{x}$ & 0 & 0 & 0 & 0 & 0 & $?$ & $e_{2, \sim}$ & $S K_{87}$ & $S K_{86}$ & $S K_{85}$ & $S K_{84}$ \\
\hline$\Delta X_{22}$ & 0 & 0 & 0 & 0 & 0 & 0 & $e_{2, \sim}$ & $80_{x}$ & $S K_{91}$ & $S K_{90}$ & $S K_{89}$ & $S K_{88}$ \\
\hline$\Delta X_{23}$ & 0 & 0 & 0 & 0 & 0 & 0 & $80_{x}$ & 0 & $S K_{95}$ & $S K_{94}$ & $S K_{93}$ & $S K_{92}$ \\
\hline$\Delta X_{24}$ & 0 & 0 & 0 & 0 & 0 & 0 & 0 & 0 & $S K_{99}$ & $S K_{98}$ & $S K_{97}$ & $S K_{96}$ \\
\hline$\Delta X_{25}$ & 0 & 0 & 0 & 0 & 0 & 0 & 0 & 0 & $S K_{103}$ & $S K_{102}$ & $S K_{101}$ & $S K_{100}$ \\
\hline$\Delta X_{26}$ & 0 & 0 & 0 & 0 & 0 & 0 & 0 & 0 & $S K_{107}$ & $S K_{106}$ & $S K_{105}$ & $S K_{104}$ \\
\hline$\Delta X_{27}$ & 0 & 0 & 0 & 0 & 0 & 0 & 0 & 0 & $S K_{111}$ & $S K_{110}$ & $S K_{109}$ & $S K_{108}$ \\
\hline$\Delta X_{28}$ & 0 & 0 & 0 & $80_{x}$ & 0 & 0 & 0 & 0 & $S K_{115}$ & $S K_{114}$ & $S K_{113}$ & $S K_{112}$ \\
\hline$\Delta X_{29}$ & 0 & $e_{2, \sim}$ & $80_{x}$ & 0 & 0 & 0 & 0 & 0 & $S K_{119}$ & $S K_{118}$ & $S K_{117}$ & $S K_{116}$ \\
\hline$\Delta X_{30}$ & $e_{2, \sim}$ & $80_{x}$ & 0 & 0 & 0 & 0 & 0 & $?$ & $S K_{123}$ & $S K_{122}$ & $S K_{121}$ & $S K_{120}$ \\
\hline$\Delta X_{31}$ & $80_{x}$ & 0 & 0 & 0 & 0 & $?$ & $?$ & $e_{0, \sim}$ & $W K_{7}$ & $W K_{6}$ & $W K_{5}$ & $W K_{4}$ \\
\hline$F T$ & $e_{0, \sim}$ & $80_{x}$ & 0 & 0 & 0 & 0 & $?$ & & & & & \\
\hline
\end{tabular}

The related-key impossible differential occurs by using the key difference $\left(\Delta M K_{15}, \Delta M K_{14}, \ldots, \Delta M K_{0}\right)=\left(80_{x}, 0, \ldots, 0\right)$. The contradiction occurs at values $X_{17,3}$ and can be shown similarly by miss in the middle manner which is given in Table 7 where the contradictory differences and the subkey bytes having nonzero differences are shown with gray background. The related-key impossible 
differential was found by imposing the difference $80_{x}$ to all 16 key bytes and observing the impossibility at the differentials. It can be concluded that the best related-key impossible differential is 22 rounds and it can not be extended by the same technique 9

Using this related-key impossible differential, we can attack up to 31 rounds of Hight. This attack covers the rounds $0-30$ and excludes the input whitening as done in 26-round impossible differential attack [19. We use the related-key impossible differential characteristic to attack 31-round of HigHT which is detailed in Table 7 The attack can be described as follows.

\section{1.) Data Collection}

(i) Choose $2^{15}$ structures of $2^{48}$ plaintexts each where the byte (2) and the least significant seven bits of the byte (3) are fixed to certain values. The bytes $(7,6,5,1,0)$ and the most significant seven bits of the byte (7) contain every possible values.

- There exist $2^{110}$ plaintext pairs in total which are encrypted by the prescribed difference in the key.

(ii) Obtain all the ciphertexts $C^{i}$ of the plaintexts $P^{i}$ encrypted with $K^{1}$ and ciphertext $C^{i^{\prime}}$ of the plaintexts $P^{i}$ encrypted with $K^{2}$ where $K^{1} \oplus K^{2}=$ $\left(80_{x}, 0, \ldots, 0\right)$. Choose only the ciphertext pairs $\left(C^{i}, C^{j^{\prime}}\right)$ satisfying the difference $\left(e_{0, \sim}, 80_{x}, 0,0,0,0, ?, ?\right)$.

- This step can be done by inserting all the ciphertexts into a hash table indexed by expected inactive bits and choosing the colliding pairs which satisfy the required difference. There is 41-bit filtering condition over the ciphertext pairs. Therefore $2^{69}$ pairs remain.

\section{2.) Filtering and Key Elimination}

We follow the steps as in 26-round attack which is given in Table 8 to reach impossible differential characteristic.

In step 24 , if a pair satisfies the impossible differential characteristic, we eliminate the corresponding guessed key. Since there is five-bit condition, each pair eliminates $2^{-5}$ of the keys and after the last pair there remain $2^{120}(1-$ $\left.2^{-5}\right)^{2^{4}} \approx 2^{119.27}$ keys.

\section{3.) Final Step:}

For every recorded 120 bit key $\left(M K_{0}, \ldots, M K_{12}, M K_{14}, M K_{15}\right)$, obtain the remaining eight bits of the key by exhaustive search by checking over three plaintext-ciphertext pairs. The probability that a wrong key is suggested is approximately $2^{-64 \times 3}=2^{-192}$. So, the expected number of wrong keys is about $2^{-192} \cdot 2^{127.27}=2^{-64.73}$. It is very likely that we can find the correct key.

${ }^{9}$ A similar attack with the same complexity can be mounted by imposing the difference $80_{x}$ to $M K_{9}$ instead of $M K_{15}$ 
Table 8. 31-Round related key impossible differential attack

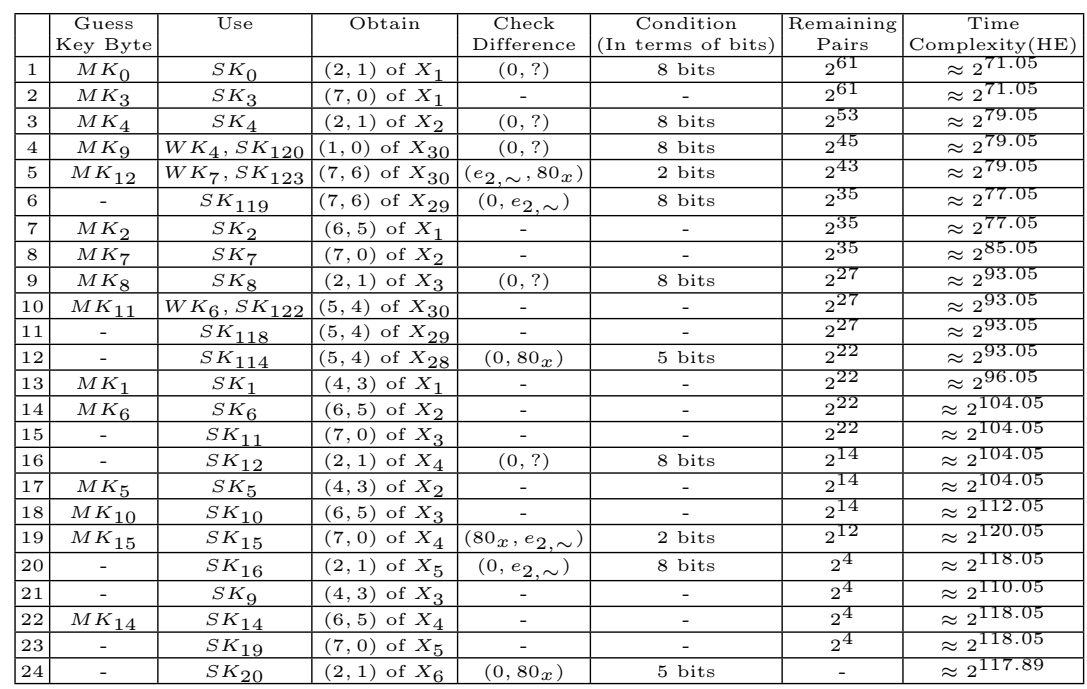

The total complexity of the steps given in Table 8 is approximately $2^{121.03}$. Since there exists $2^{119.27}$ remaining keys, the complexity of the final step is approximately $2^{127.27}$. Therefore, the complexity of this whole attack is $2^{127.28}$ 31-round Hight computations. Even if this attack is not significant compared to the exhaustive search, it is still an important result against HiGHT which reduces the safety margin of HigHT to one round.

\section{Conclusion}

In this paper, we present the first related-key cryptanalysis of PRESENT and improve the recent impossible differential attacks on HigHT. Although our attacks are totally theoretical and have no practical implications, they show new results for both ciphers. The related-key attacks on Present can be seen as a new approach to see the security of the cipher. HiGHT, on the other hand, was shown to be secure up to 19 rounds in the original proposal and recently attacked up to 28 rounds. However, our results show that the security of HigHT can be further reduced up to one round.

\section{Acknowledgements}

The authors would like to thank anonymous reviewers of ACISP 2009 for pointing out recent results on PRESENT and Jonsung Kim for the additional information about Hight. We also like to thank Meltem Sönmez Turan, Shahram Khazaei and Martijn Stam for reviewing the previous versions of the paper. 
The simulations were run on the HPC Cluster of the Department of Computer Engineering, Middle East Technical University.

\section{References}

1. Joan Daemen and Vincent Rijmen. The Design of Rijndael. Springer-Verlag New York, Inc., Secaucus, NJ, USA, 2002.

2. Deukjo Hong, Jaechul Sung, Seokhie Hong, Jongin Lim, Sangjin Lee, Bonseok Koo, Changhoon Lee, Donghoon Chang, Jaesang Lee, Kitae Jeong, Hyun Kim, Jongsung Kim, and Seongtaek Chee. HIGHT: A New Block Cipher Suitable for Low-Resource Device. In Louis Goubin and Mitsuru Matsui, editors, CHES, volume 4249 of Lecture Notes in Computer Science, pages 46-59. Springer, 2006.

3. South Korea Telecommunications Technology Associations (TTA). 64-bit Block Cipher HIGHT. Standardization Number TTAS.KO-12.0040, 27 December 2006.

4. Andrey Bogdanov, Lars R. Knudsen, Gregor Leander, Christof Paar, Axel Poschmann, Matthew J. B. Robshaw, Yannick Seurin, and C. Vikkelsoe. PRESENT: An Ultra-Lightweight Block Cipher. In Pascal Paillier and Ingrid Verbauwhede, editors, CHES, volume 4727 of Lecture Notes in Computer Science, pages 450-466. Springer, 2007.

5. Chae Hoon Lim and Tymur Korkishko. mCrypton - A Lightweight Block Cipher for Security of Low-Cost RFID Tags and Sensors. In JooSeok Song, Taekyoung Kwon, and Moti Yung, editors, WISA, volume 3786 of Lecture Notes in Computer Science, pages 243-258. Springer, 2005.

6. François-Xavier Standaert, Gilles Piret, Neil Gershenfeld, and Jean-Jacques Quisquater. SEA: A Scalable Encryption Algorithm for Small Embedded Applications. In Josep Domingo-Ferrer, Joachim Posegga, and Daniel Schreckling, editors, CARDIS, volume 3928 of Lecture Notes in Computer Science, pages 222236. Springer, 2006.

7. Matthew J. B. Robshaw. Searching for Compact Algorithms: CGEN. In Phong Q. Nguyen, editor, VIETCRYPT, volume 4341 of Lecture Notes in Computer Science, pages 37-49. Springer, 2006.

8. Gregor Leander, Christof Paar, Axel Poschmann, and Kai Schramm. New Lightweight DES Variants. In Alex Biryukov, editor, FSE, volume 4593 of Lecture Notes in Computer Science, pages 196-210. Springer, 2007.

9. David J. Wheeler and Roger M. Needham. TEA, a Tiny Encryption Algorithm. In Bart Preneel, editor, FSE, volume 1008 of Lecture Notes in Computer Science, pages 363-366. Springer, 1994.

10. David J. Wheeler and Roger M. Needham. TEA Extensions. October 1997.

11. The eSTREAM Portfolio. The eSTREAM Project, http://www.ecrypt.eu.org/ stream/, September 2008.

12. Meiqin Wang. Differential Cryptanalysis of Reduced-Round PRESENT. In Serge Vaudenay, editor, AFRICACRYPT, volume 5023 of Lecture Notes in Computer Science, pages 40-49. Springer, 2008.

13. Martin Albrecht and Carlos Cid. Algebraic Techniques in Differential Cryptanalysis. To appear in proceedings of FSE, 2009.

14. Muhammad Reza Z'aba, Håvard Raddum, Matthew Henricksen, and Ed Dawson. Bit-Pattern Based Integral Attack. In Kaisa Nyberg, editor, FSE, volume 5086 of Lecture Notes in Computer Science, pages 363-381. Springer, 2008. 
15. Baudoin Collard and François-Xavier Standaert. A Statistical Saturation Attack against the Block Cipher PRESENT . To appear in proceedings of CT-RSA, 2009.

16. Alex Biryukov and David Wagner. Slide Attacks. In Knudsen [26], pages 245-259.

17. Eli Biham. New Types of Cryptanalytic Attacks Using Related Keys. Journal of Cryptology, 7(4):229-246, 1994.

18. Jiqiang Lu. Cryptanalysis of Block Ciphers. PhD thesis, Royal Holloway, University of London, England, July 2008.

19. Jiqiang Lu. Cryptanalysis of Reduced Versions of the HIGHT Block Cipher from CHES 2006. In Kil-Hyun Nam and Gwangsoo Rhee, editors, ICISC, volume 4817 of Lecture Notes in Computer Science, pages 11-26. Springer, 2007.

20. Eli Biham, Orr Dunkelman, and Nathan Keller. Related-Key Boomerang and Rectangle Attacks. In Ronald Cramer, editor, EUROCRYPT, volume 3494 of Lecture Notes in Computer Science, pages 507-525. Springer, 2005.

21. Eli Biham, Orr Dunkelman, and Nathan Keller. New Combined Attacks on Block Ciphers. In Henri Gilbert and Helena Handschuh, editors, FSE, volume 3557 of Lecture Notes in Computer Science, pages 126-144. Springer, 2005.

22. Eli Biham, Orr Dunkelman, and Nathan Keller. The Rectangle Attack - Rectangling the Serpent. In Birgit Pfitzmann, editor, EUROCRYPT, volume 2045 of Lecture Notes in Computer Science, pages 340-357. Springer, 2001.

23. Eli Biham, Alex Biryukov, and Adi Shamir. Cryptanalysis of Skipjack Reduced to 31 Rounds Using Impossible Differentials. Journal of Cryptology, 18(4):291-311, 2005.

24. Eli Biham, Alex Biryukov, and Adi Shamir. Miss in the Middle Attacks on IDEA and Khufu. In Knudsen [26], pages 124-138.

25. Orr Dunkelman. Techniques for Cryptanalysis of Block Ciphers. PhD thesis, Technion, Israel, February 2006.

26. Lars R. Knudsen, editor. Fast Software Encryption, 6th International Workshop, FSE '99, Rome, Italy, March 24-26, 1999, Proceedings, volume 1636 of Lecture Notes in Computer Science. Springer, 1999.

\section{A A Brief Description of Related-Key Rectangle Attack}

Let $E$ denote the encryption function of the attacked cipher which is treated as a cascade of four subciphers as $E=E_{f} \circ E_{1} \circ E_{0} \circ E_{b}$ where the core $E^{\prime}=E_{1} \circ E_{0}$ is covered by additional rounds called $E_{b}$ and $E_{f}$ which are the subciphers before and after the core function respectively to be used in key recovery. The relatedkey differential is the quadruple $(\Delta X, \Delta Y, \Delta K, p)$ satisfying following under the nonlinear $K$-keyed function $F_{K}$,

$$
\operatorname{Pr}\left[F_{K}(P) \oplus F_{K \oplus \Delta K}(P \oplus \Delta X)=\Delta Y\right]=p .
$$

Here, $\Delta X$ and $\Delta Y$ are the corresponding input and output differences respectively, $\Delta K$ the key difference and $p$ is the corresponding probability. We say the related-key differential characteristics $\Delta X \stackrel{\Delta K}{\rightarrow} \Delta Y$ holds with probability $\operatorname{Pr}=p$.

Let $\alpha \stackrel{\Delta K^{12}}{\longrightarrow} \beta$ with probability $p$ be the first related-key differential used for $E_{0}$ and $\gamma \stackrel{\Delta K^{13}}{\longrightarrow} \delta$ with probability $q$ be the second differential used for 
$E_{1}$. Here, $(\alpha, \beta)$ and $(\gamma, \delta)$ stand for the input-output differences for $E_{0}$ and $E_{1}$ respectively. We define $\hat{p}$ and $\hat{q}$ as the probabilities related to $\alpha$ and $\delta$ respectively as follows: $\hat{p}=\sqrt{\sum_{\beta} P_{\Delta K^{12}}^{2}(\alpha \rightarrow \beta)}$ and $\hat{q}=\sqrt{\sum_{\gamma} P_{\Delta K^{13}}^{2}(\gamma \rightarrow \delta)}$. Here, $\beta$ and $\gamma$ are the possible differences at the end of $E_{0}$ and at the beginning of $E_{1}$. The key differences $\Delta K^{12}=K^{1} \oplus K^{2}=K^{3} \oplus K^{4}$ and $\Delta K^{13}=K^{1} \oplus K^{3}=K^{2} \oplus K^{4}$ are the respective key differences for the subciphers $E_{0}$ and $E_{1}$. The subciphers before and after the core function are formed according to the $\alpha$ and $\delta$ differences. The basic related-key rectangle attack for the core function works as follows:

- Take a randomly chosen plaintext $P_{1}$ and form $P_{2}=P_{1} \oplus \alpha$.

- Obtain the corresponding ciphertexts $C_{1}=E_{K^{1}}^{\prime}\left(P_{1}\right)$ and $C_{2}=E_{K^{2}}^{\prime}\left(P_{2}\right)$, where $K^{2}=K^{1} \oplus \Delta K^{12}$.

- Pick another randomly chosen plaintext $P_{3}$ and form $P_{4}=P_{3} \oplus \alpha$.

- Obtain the corresponding ciphertexts $C_{3}=E_{K^{3}}^{\prime}\left(P_{3}\right)$ and $C_{4}=E_{K^{4}}^{\prime}\left(P_{4}\right)$, where $K^{3}=K^{1} \oplus \Delta K^{13}$ and $K^{4}=K^{3} \oplus \Delta K^{12}$.

- Check $C_{1} \oplus C_{3}=C_{2} \oplus C_{4}=\delta$.

The probability of the rectangle distinguisher is given by $\operatorname{Pr}=2^{-n} \hat{p}^{2} \hat{q}^{2}$ where $n$ is the block size. If $\hat{p} \cdot \hat{q}$ is sufficiently high, the rectangle distinguisher works. As shown in [25], if the expected number of right quartets is taken to be four, then there is at least one right quartet in the data set with probability 0.982 since it is a Poisson distribution with expectation of four. Therefore, for this success rate, the number of plaintext pairs needed is $N=2^{n / 2+1} / \hat{p} \hat{q}$ that consist of $2^{n+2} / \hat{p}^{2} \hat{q}^{2}$ quartets expecting four right quartets at a time. Further details about rectangle and boomerang attacks can be found in [20|21|22 25.

\section{B Key Schedule Properties of HIGHT}

Table 9. Relations of the original key with whitening keys and subkeys

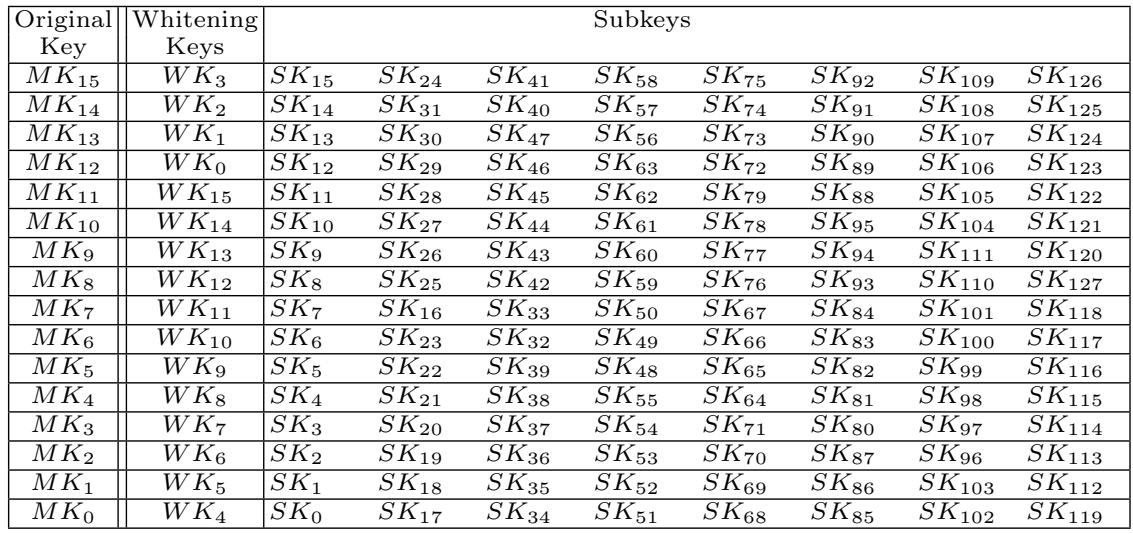

